\title{
Usefulness of waist-to-height ratio in screening incident hypertension among Japanese community-dwelling middle-aged and elderly individuals
}

Ryuichi Kawamoto ${ }^{1,2^{*}}$, Asuka Kikuchi ${ }^{1,2}$, Taichi Akase ${ }^{1,2}$, Daisuke Ninomiya ${ }^{1,2}$ and Teru Kumagi ${ }^{1}$

\begin{abstract}
Background: The incidence of hypertension is increasing worldwide and obesity is one of the most significant risk factors. Obesity can be defined by various anthropometric indices such as body mass index (BMI), waist-to-hip ratio (WHpR), and waist-to-height ratio (WHtR). This study examined a range of anthropometric indices and their relationships with hypertension.

Methods: This study included 768 men aged $70 \pm 10$ years and 959 women aged $70 \pm 8$ years from a rural village. The relationship between anthropometric indices (BMI, WHpR, and WHtR) and hypertension was examined using cross-sectional (baseline, $N=1727$ ) and cohort data (follow-up, $N=419)$. Receiver operating characteristic (ROC) analysis was used to determine the predictive ability of obesity indices for hypertension in both genders. Logistic regression models were used to evaluate WHtR as a significant predictor of hypertension.

Results: In the cross-sectional study, WHtR, BMI, and WHpR showed significant predictive abilities for hypertension in both genders, with WHtR showing the strongest predictive ability. Also, in the cohort study, WHtR showed a significant predictive ability for incident hypertension in both genders, and, for women, BMI as well as WHtR had also predictive ability. In the cross-sectional study, the optimal WHtR cutoff values were 0.53 (sensitivity, 44.3\%; specificity, 80.2\%) for men and 0.54 (sensitivity, 60.9\%; specificity, 68.6\%) for women. In the cohort study, the optimal WHtR values were 0.47 (sensitivity, 85.4\%; specificity, 39.8\%) for men and 0.51 (sensitivity, 66.7\%; specificity, 58.2\%) for women.
\end{abstract}

Conclusions: The results suggest that WHtR is a useful screening tool for hypertension among Japanese middleaged and elderly community-dwelling individuals.

Keywords: Waist-to-height ratio, Hypertension, Anthropometric indices, Community-dwelling individuals

\footnotetext{
* Correspondence: rykawamo@m.ehime-u.ac.jp

'Department of Community Medicine, Ehime University Graduate School of Medicine, Shitsukawa, Toon-city, Japan

${ }^{2}$ Department of Internal Medicine, Seiyo Municipal Nomura Hospital, 9-53

Nomura, Nomura-cho, Seiyo-City, Japan
}

(c) The Author(s). 2020 Open Access This article is licensed under a Creative Commons Attribution 4.0 International License, which permits use, sharing, adaptation, distribution and reproduction in any medium or format, as long as you give appropriate credit to the original author(s) and the source, provide a link to the Creative Commons licence, and indicate if changes were made. The images or other third party material in this article are included in the article's Creative Commons licence, unless indicated otherwise in a credit line to the material. If material is not included in the article's Creative Commons licence and your intended use is not permitted by statutory regulation or exceeds the permitted use, you will need to obtain permission directly from the copyright holder. To view a copy of this licence, visit http://creativecommons.org/licenses/by/4.0/ The Creative Commons Public Domain Dedication waiver (http://creativecommons.org/publicdomain/zero/1.0/) applies to the data made available in this article, unless otherwise stated in a credit line to the data. 


\section{Background}

The incidence of hypertension is increasing worldwide with the continuous increase in obesity prevalence [1]. Since obesity increases the risk of hypertension, addressing the obesity and hypertension epidemic is crucial [2]. Obesity can be defined by various obesity-related anthropometric indices such as body mass index (BMI), waist circumference (WC), waist-to-height ratio (WHtR), and waist-to-hip ratio (WHpR). The World Health Organization recommends the use of some anthropometric parameters as screening markers for individuals at risk for cardiovascular disease (CVD) as they can be determined easily and inexpensively [3] Epidemiological studies have shown that these anthropometric indices predict incident hypertension [4-9]. BMI is the most widely used indicator of obesity, but it does not reflect central fat distribution $[10,11]$. In Japan, cross-sectional and prospective studies have demonstrated a strong association between WHtR and hypertension $[4,5,12]$. However, which index best predicts the development of hypertension remains controversial [13-16], and more specifically, there is a lack of consensus on the best predictive indicator of hypertension among Japanese middle-aged and elderly individuals.

To address this controversy, we investigated the relationship between baseline visceral obesity indices (BMI, WHpR, and WHtR) and potential risk factors and hypertension using cross-sectional and prospective cohort data from community-dwelling middle-aged and elderly individuals.

\section{Methods \\ Study participants and data collection}

This study enrolled a population-based sample of community-dwelling Japanese adults from the Nomura Health and Welfare Center in a rural town in the Ehime prefecture of Japan. Factors for increased CVD risk were examined from annual health checkup data [17]. Follow-up assessment cycles are performed every 3 years.

Overall, 1832 community-dwelling participants aged 20-95 years (818 men and 1014 women) were enrolled between April and November 2014. In the present study, the analysis was restricted to participants aged $\geq 40$ years without any missing baseline data. The initial dataset consisted of 1727 participants (768 men and 959 women) aged 40-95 years, on whom the follow-up survey, spanning 3 years, was performed. Of these participants, 1308 were excluded due to the presence of hypertension $(N=1119)$ and missing data $(N=189)$, especially data on WC and blood pressure. The final dataset comprised 419 non-hypertensive participants (164 men and 255 women). Figure 1 shows a flowchart describing the inclusion of participants.

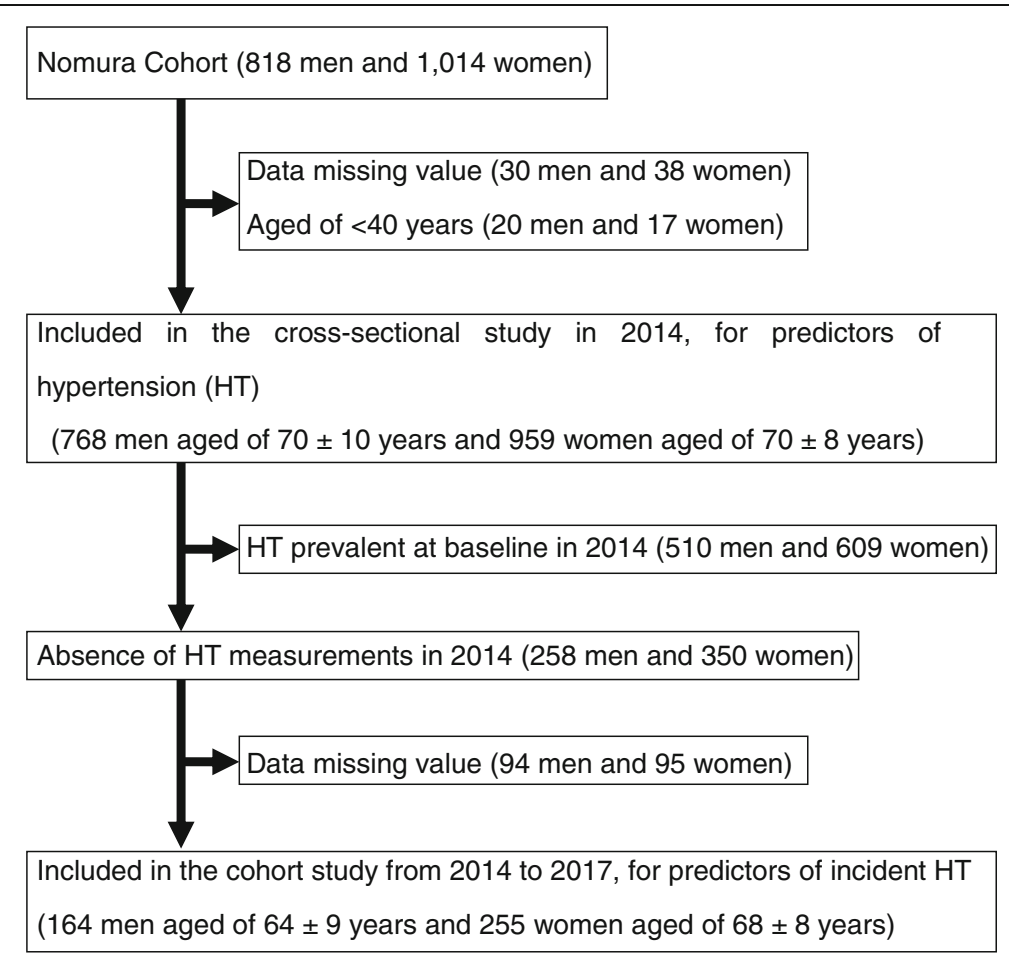

Fig. 1 Flowchart. For the cross-sectional analyses, data from the 2014 cycle $(n=1727)$ were used. For the longitudinal analyses, only participants in whom hypertension was not prevalent at baseline in 2014 were included $(n=419)$ 
All participants provided written informed consent, and the Nomura study was approved by the Ehime University Medical School Ethics Committee. All procedures performed in the study involving human participants were in accordance with the ethical standards of the institutional research committee in which the study was conducted (IRB Approval number: 1402009).

\section{Anthropometric and laboratory measurements}

Baseline anthropometric indices such as BMI, WC, WHpR, and WHtR were measured. BMI was calculated by dividing weight in kilograms $(\mathrm{kg})$ by height in meters squared $\left(\mathrm{m}^{2}\right)$. WC was measured in the horizontal plane at the mid-point between the anterior iliac crest and the inferior margin of the rib. WHtR was calculated as WC $(\mathrm{cm}) /$ height $(\mathrm{cm})$. WHpR was calculated as WC $(\mathrm{cm}) /$ hip circumference $(\mathrm{cm})$. In addition, lifestyle-related factors such as smoking, alcohol consumption, and regular exercise habits were also investigated by individual interviews conducted using a structured questionnaire. Smoking habits were determined by multiplying the number of years the person has smoked by the average number of packs smoked per day (pack year), and participants were classified as never smokers, former smokers, light smokers $(<30$ pack year), and heavy smokers $(\geq 30$ pack year) [18]. Alcohol consumption was measured using the traditional Japanese unit of alcohol, go, which is equivalent to $22.9 \mathrm{~g}$ of ethanol, and the participants were classified as non-drinkers, occasional drinkers $(<1$ unit/day), daily light drinkers ( $<2$ unit/day), and daily heavy drinkers ( $\geq 2$ unit/day) [19]. Systolic blood pressure (SBP) and diastolic blood pressure (DBP) were measured twice using an automatic oscillometric blood pressure recorder, on the right upper arm of the subjects with an appropriate-sized cuff in the sedentary position after having rested for at least $5 \mathrm{~min}$. The two values were then averaged.

For all these individuals, triglycerides (TG), highdensity lipoprotein cholesterol (HDL-C), low-density lipoprotein cholesterol (LDL-C), hemoglobin A1c (HbA1c), serum uric acid (SUA), and creatinine $(\mathrm{Cr})$ were measured during an overnight fast of over $11 \mathrm{~h}$. The estimated glomerular filtration ratio (eGFR) was calculated using the Chronic Kidney Disease Epidemiology Collaboration (CKD-EPI) equations modified by the Japanese coefficient (eGFR CKDEPI $_{\text {): }}$ Male, $\mathrm{Cr} \leq 0.9 \mathrm{mg} / \mathrm{dl}$, $141 \times(\mathrm{Cr} / 0.9){ }^{-0.411} \times 0.993{ }^{\text {age }} \times 0.813 ; \mathrm{Cr}>0.9 \mathrm{mg} / \mathrm{dl}$, $141 \times(\mathrm{Cr} / 0.9)^{-1.209} \times 0.993$ age $\times 0.813$; Female, $\mathrm{Cr} \leq 0.7$ $\mathrm{mg} / \mathrm{dl}, 144 \times(\mathrm{Cr} / 0.7)^{-0.329} \times 0.993^{\text {age }} \times 0.813 ; \mathrm{Cr}>0.7$ $\mathrm{mg} / \mathrm{dl}, 144 \times(\mathrm{Cr} / 0.7)^{-1.209} \times 0.993^{\text {age }} \times 0.813$ [20].

\section{Criteria for clinical diagnosis of hypertension}

Normotension was defined as not being on antihypertensive medication and having a SBP $<120 \mathrm{mmHg}$ and
DBP $<80 \mathrm{mmHg}$. Prehypertension was defined as not being on antihypertensive medication and having a SBP of 120 to $139 \mathrm{mmHg}$ and/or DBP 80 to $89 \mathrm{mmHg}$. Hypertension was defined as being on antihypertensive medication and/or having SBP $\geq 140 \mathrm{mmHg}$ and/or DBP $\geq 90 \mathrm{mmHg}$ according to the definitions of the Joint $\mathrm{Na}$ tional Committee 7 [21].

\section{Statistics}

All the data were analyzed using the IBM Statistical Package for Social Sciences version 21.0 (IBM SPSS Statistics Inc. Chicago IL. USA). Continuous variables were presented as means \pm standard deviation (SD) if the data were normally distributed and as medians (interquartile ranges) if the distributions were skewed (e.g., TG and HbA1c). Subjects were divided into two groups based on gender, and differences among the groups were analyzed by Student's t-test for continuous variables or a chisquared $\left(x^{2}\right)$ test for categorical variables. For all analyses, parameters with non-normal distributions were used after log-transformation. Multiple logistic regression analysis was used to evaluate the contribution of the baseline WHtR and confounding factors (i.e., gender, age, smoking status, drinking status, exercise habits, presence of CVD, TG, HDL-C, LDL-C, use of antidyslipidemic medication, HbAlc, use of antidiabetic medication, eGFR, and SUA) on the prevalence of hypertension in the cross-sectional study and on the incidence of hypertension in the cohort study. If any independent variables are correlated with each other $(r \geq 0.6)$ (known as multicollinearity) the variable was removed from the multivariate analysis (e.g., BMI, WHtR, and WHpR). In addition, areas under the receiver operating characteristic (ROC) curves were determined for each variable to identify the predictors of hypertension. A ROC curve is a plot of sensitivity (true positive) versus 1-specificity (false positive) for different cutoff points of a parameter. Area under the ROC curve (AUC) is a summary of the overall diagnostic accuracy of the test including standard errors. Predictive values were calculated as sensitivity/ [sensitivity+(1-specificity)] (positive predictive value) and specificity/[(1-sensitivity) + specificity] (negative predictive value). The optimal cutoff values were defined as the point at which the value of sensitivity + specificity -1 was maximum (Youden's index) [22]. A $p$-value $<0.05$ was considered significant.

\section{Results}

Baseline characteristics of study subjects categorized by presence of hypertension in the cross-sectional and cohort studies

A total of 1727 participants were included at baseline in this cross-sectional study. Baseline characteristics of the subjects categorized by hypertension status are shown in 
Table 1. The study included 768 men aged $70 \pm 10$ (range, 40-95) years and 959 women aged $70 \pm 8$ (range, $41-90)$ years. The proportion of men was $42.4 \%$ in the normotension group and $45.6 \%$ in the hypertension group. Age, BMI, WC, WHpR, WHtR, smoking habit, prevalence of CVD, SBP, DBP, use of antihypertensive medication, TG, use of antidyslipidemic medication, HbA1c, use of antidiabetic medication, and SUA were significantly higher in the hypertension group, but HDL$\mathrm{C}$, LDL-C, and eGFR were significantly lower. There were no differences in gender, drinking status, or exercise habits. In the cohort study as shown in Table 2, the proportion of men was $38.0 \%$ in the normotension group and $43.2 \%$ in the hypertension group. Age, BMI, WC, WHtR WHpR, SBP, DBP, and SUA were significantly higher in the hypertension group, but prevalence of exercise habits was significantly lower.

\section{Results of the ROC curve analyses to identify optimal} obesity indices to distinguish subjects with hypertension in the cross-sectional and cohort studies

Figure 2 shows the AUC for BMI, WHpR, and WHtR for hypertension in both genders using ROC analyses. In the cross-sectional study, WHtR, BMI, and WHpR showed significant predictive ability for incident hypertension in both genders. Also, in the cohort study, WHtR showed a significant predictive ability for incident hypertension in both genders, and, for women, BMI as well as WHtR had also predictive ability. As shown in Table 3, in men WHtR showed significantly stronger predictive ability than WHpR and BMI, but in women WHtR as well as BMI were stronger than WHpR. In cohort study, there was no any difference of three devices.

\section{Odds ratios and $95 \%$ confidence interval $(\mathrm{CI})$ for hypertension by quartile of WHtR in the cross-sectional and cohort studies}

To further investigate whether WHtR can explain hypertension independently of other confounding factors, a multiple logistic regression analysis was performed. Hypertension was the dependent variable and various confounding factors (e.g., age, smoking status, drinking status, exercise habits, presence of CVD, SBP, DBP, use of antihypertensive medication, TG, HDL-C, LDL-C, use of antidyslipidemic medication, SUA, and eGFR) were the explanatory variables (Table 4 ). In both the cross-

Table 1 Baseline characteristics of subjects according to hypertension status in the cross-sectional study

\begin{tabular}{|c|c|c|c|}
\hline & Normotension & Hypertension & $\boldsymbol{P}$-value* \\
\hline Baseline Characteristics & $N=608$ & $\boldsymbol{N}=1119$ & \\
\hline Gender (male, \%) & $258(42.4)$ & $510(45.6)$ & 0.224 \\
\hline Age (years) & $66 \pm 9$ & $72 \pm 8$ & $<0.001$ \\
\hline BMI $\left(\mathrm{kg} / \mathrm{m}^{2}\right)$ & $21.8 \pm 2.8$ & $23.4 \pm 3.2$ & $<0.001$ \\
\hline Waist circumference (cm) & $78.9 \pm 8.0$ & $82.7 \pm 8.9$ & $<0.001$ \\
\hline WHtR & $0.51 \pm 0.05$ & $0.54 \pm 0.06$ & $<0.001$ \\
\hline WHpR & $0.88 \pm 0.06$ & $0.91 \pm 0.06$ & $<0.001$ \\
\hline Smoking habit (never/past/light/heavy (\%)) & $71.2 / 15.5 / 5.3 / 8.1$ & $72.4 / 20.5 / 1.7 / 5.5$ & $<0.001$ \\
\hline Drinking status (never/occasional/light/heavy (\%)) & $50.5 / 25.2 / 8.7 / 15.6$ & $50.3 / 20.6 / 10.3 / 18.9$ & 0.069 \\
\hline Exercise habits (\%) & 36.0 & 38.0 & 0.435 \\
\hline Cardiovascular disease (\%) & 4.6 & 8.0 & 0.009 \\
\hline Systolic blood pressure (mmHg) & $122 \pm 12$ & $144 \pm 15$ & $<0.001$ \\
\hline Diastolic blood pressure $(\mathrm{mmHg})$ & $72 \pm 8$ & $81 \pm 10$ & $<0.001$ \\
\hline Antihypertensive medication (\%) & 0 & 68.7 & $<0.001$ \\
\hline Triglycerides (mg/dl) & $83(61-114)$ & $91(69-129)$ & $<0.001$ \\
\hline HDL cholesterol (mg/dl) & $68 \pm 18$ & $64 \pm 16$ & $<0.001$ \\
\hline LDL cholesterol (mg/dl) & $122 \pm 29$ & $119 \pm 30$ & 0.047 \\
\hline Antidyslipidemic medication (\%) & 14.3 & 26.5 & $<0.001$ \\
\hline Hemoglobin A1c (\%) & $5.6(5.4-5.8)$ & $5.7(5.5-6.0)$ & $<0.001$ \\
\hline Antidiabetic medication (\%) & 5.6 & 10.5 & $<0.001$ \\
\hline Serum uric acid (mg/dL) & $5.1 \pm 1.3$ & $5.4 \pm 1.4$ & $<0.001$ \\
\hline Estimated GFR (ml/min/1.73 m²/year) & $74.9 \pm 9.8$ & $69.6 \pm 12.2$ & $<0.001$ \\
\hline
\end{tabular}

$B M I$ body mass index; WHtR waist/height ratio; WHpR waist/hip ratio; HDL high-density lipoprotein; $L D L$ low-density lipoprotein; GFR glomerular filtration ratio. Data presented are mean \pm standard deviation. Data for triglycerides and hemoglobin A1c is skewed, and presented as median (interquartile range) values. * $P$ value: Student's t-test for the continuous variables or the $x^{2}$ test for the categorical variables. Bold values indicate significance $(p<0.05)$ 
Table 2 Baseline characteristics of subjects according to hypertension status in the cohort study

\begin{tabular}{|c|c|c|c|}
\hline & Normotension & Hypertension & $P$-value* \\
\hline Baseline Characteristics & $\boldsymbol{N}=324$ & $\boldsymbol{N}=95$ & \\
\hline Gender (male, \%) & $123(38.0)$ & $41(43.2)$ & 0.403 \\
\hline Age (years) & $65 \pm 9$ & $67 \pm 9$ & 0.036 \\
\hline BMI $\left(\mathrm{kg} / \mathrm{m}^{2}\right)$ & $21.4 \pm 2.6$ & $22.5 \pm 2.7$ & $<0.001$ \\
\hline Waist circumference (cm) & $78.0 \pm 7.7$ & $80.5 \pm 7.5$ & 0.005 \\
\hline WHtR & $0.50 \pm 0.05$ & $0.52 \pm 0.06$ & 0.001 \\
\hline WHpR & $0.87 \pm 0.06$ & $0.89 \pm 0.06$ & 0.017 \\
\hline Smoking habit (never/past/light/heavy (\%)) & 71.9/15.1/4.6/8.3 & $74.7 / 12.6 / 5.3 / 7.4$ & 0.910 \\
\hline Drinking status (never/occasional/light/heavy (\%)) & $51.5 / 25.3 / 7.1 / 16.0$ & $43.2 / 25.3 / 10.5 / 21.1$ & 0.361 \\
\hline Exercise habits (\%) & 38.3 & 26.3 & 0.038 \\
\hline Cardiovascular disease (\%) & 3.1 & 3.2 & 1.000 \\
\hline Systolic blood pressure (mmHg) & $119 \pm 11$ & $130 \pm 8$ & $<0.001$ \\
\hline Diastolic blood pressure (mmHg) & $71 \pm 8$ & $77 \pm 7$ & $<0.001$ \\
\hline Antihypertensive medication (\%) & 0 & 0 & 1.000 \\
\hline Triglycerides (mg/dl) & $81(59-109)$ & $88(63-126)$ & 0.285 \\
\hline HDL cholesterol (mg/dl) & $69 \pm 17$ & $67 \pm 19$ & 0.498 \\
\hline LDL cholesterol (mg/dl) & $123 \pm 29$ & $120 \pm 28$ & 0.390 \\
\hline Antidyslipidemic medication (\%) & 16.0 & 15.8 & 1.000 \\
\hline Hemoglobin A1c (\%) & $5.6(5.4-5.8)$ & $5.6(5.4-5.8)$ & 0.494 \\
\hline Antidiabetic medication (\%) & 4.6 & 3.2 & 0.774 \\
\hline Serum uric acid (mg/dL) & $5.0 \pm 1.3$ & $5.4 \pm 1.3$ & 0.002 \\
\hline Estimated GFR (ml/min/1.73 m²/year) & $76.0 \pm 10.0$ & $75.0 \pm 8.0$ & 0.361 \\
\hline
\end{tabular}

Data presented are mean \pm standard deviation. Data for triglycerides and hemoglobin A1c is skewed, and presented as median (interquartile range) values. * $P$ value: Student's t-test for the continuous variables or the $x^{2}$ test for the categorical variables. Bold values indicate significance $(p<0.05)$

sectional and cohort studies, increased WHtR showed an increasing trend with increased prevalence and incidence of hypertension.

\section{Best cutoff values of WHtR to predict hypertension in the cross-sectional and cohort studies}

In the cross-sectional study, the optimal WHtR cutoff values for predicting hypertension were 0.53 (sensitivity, 44.3\%; specificity, $80.2 \%$ ) for men and 0.54 (sensitivity, $60.98 \%$; specificity, 68.6\%) for women (Table 5). In the cohort study, the optimal WHtR values were 0.47 (sensitivity, $85.4 \%$; specificity, $39.8 \%$ ) for men and 0.51 (sensitivity, 66.7\%; specificity, 58.2\%) for women.

\section{Discussion}

In the present study, WHtR was significantly and independently associated with the prevalence of hypertension in this cross-sectional study and the incidence of hypertension in this cohort study. The mean AUC of the WHtR (men, $\mathrm{AUC}=0.613 ;$ 95\% CI, 0.515-0.710, $p=0.031$; women, $\mathrm{AUC}=0.619 ; 95 \% \mathrm{CI}, 0.536-0.702, p=0.007)$ was higher than that of the BMI (men, $\mathrm{AUC}=0.578 ; 95 \% \mathrm{CI}$, $0.483-0.674, p=0.133$; women, $\mathrm{AUC}=0.631 ; 95 \% \mathrm{CI}$, $0.546-0.716, p=0.003$ ) and WHpR (men, AUC =0.600;
95\% CI, 0.499-0.701, $p=0.056$; women, $\mathrm{AUC}=0.579$; 95\% CI, 0.495-0.662, $p=0.076)$ which are conventional obesity indices among both genders. To the best of our knowledge, few epidemiologic studies have quantified the relevance of WHtR in predicting incident hypertension in Japanese middle-aged and elderly community-dwelling individuals.

A variety of anthropometric indicators have been developed to identify CVD risk factors such as hypertension, diabetes, and dyslipidemia [23]. To date, however, no definitive measurement tool has been developed for the prediction of hypertension [7]. This study examined three different indicators of fat distribution-BMI, WHpR, and WHtR - which have reportedly been associated with the prevalence of hypertension in both genders. In previous cross-sectional studies, ROC curve analyses demonstrated that WHtR was a better indicator than other indices among young men and women, but BMI and WC were more sensitive markers among middle-aged men and women [16]. Meanwhile, for middle-aged and elderly Brazilians, Dutra et al. [8] reported that WC and WHpR had a stronger relationship with prevalence of hypertension compared to other indices. Population-based prospective studies among Japanese men and women showed that 


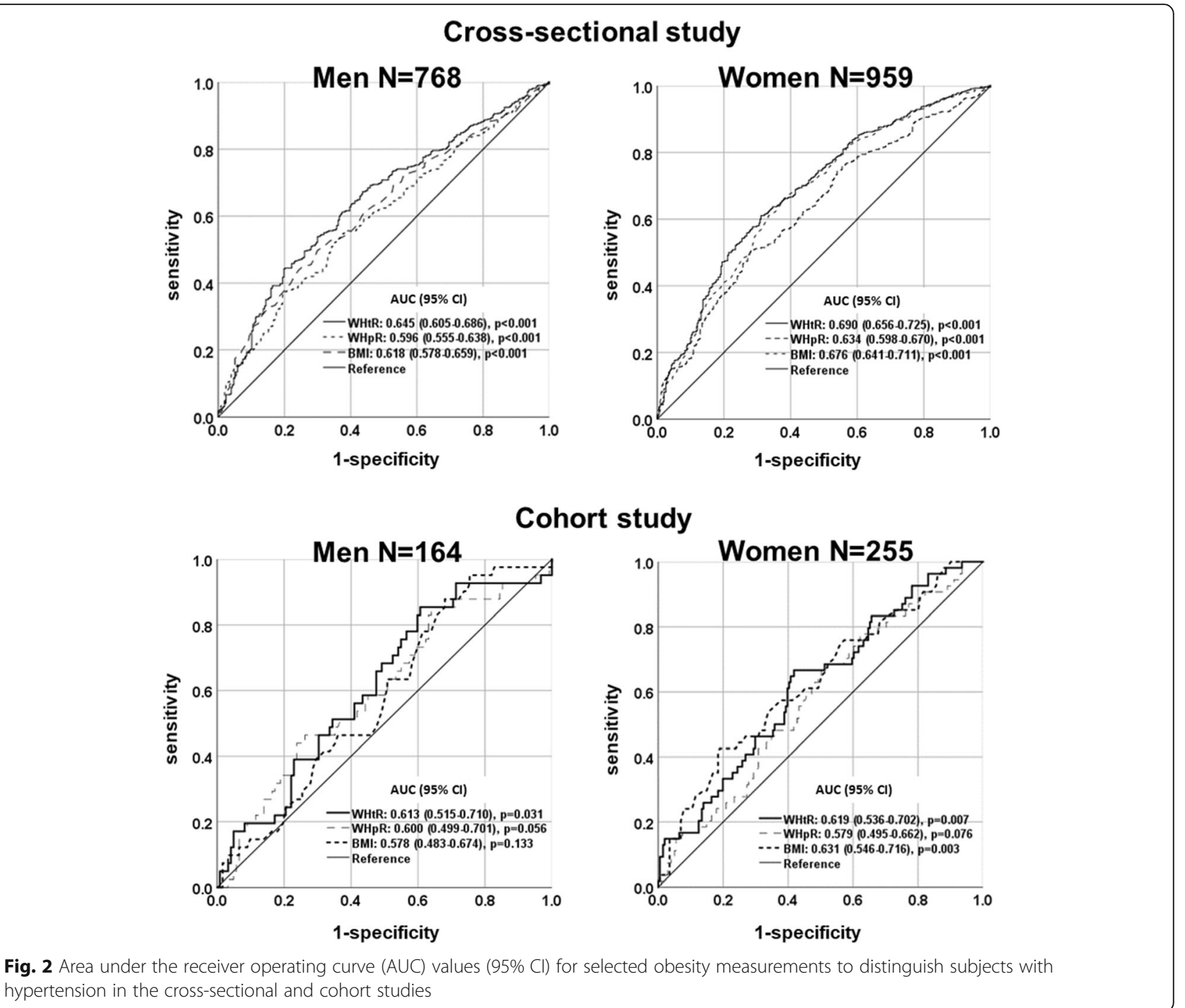

both BMI and WC were significant predictors for hypertension [4]. In meta-analyses involving 309,585 participants (men: $51.6 \pm 9.6$ years; women: $51.0 \pm 9.3$ years), WHtR had the strongest prediction ability for hypertension, which was also confirmed in subgroup analyses based on gender and country [9]. Also, in our Japanese middle-aged and elderly participants, an increasing WHtR was found to be a significant indicator for the incidence of hypertension, and in women high BMI also have the highest correlation with incident hypertension. This result may be due to the following two reasons. First, WHR can distinguish central obesity from lower body and general obesity and carries some information on both overall obesity and abdominal obesity, since WHtR is positively correlated with BMI and WC. Second, the explanation for this finding might be the difference in body composition by gender [24]. Men tend to have greater skeletal muscles than women and women tend to have a higher percentage of body fat than men [25]. Thus, the inconsistent association of obesity indices and hypertension may be due to different races, age groups, and sexes [16].

The mechanisms that lead to increased incidence of hypertension in individuals with increased WHtR remain to be elucidated. BMI is the most common anthropometric index and is strongly related to body fat, but is not necessarily related to abdominal obesity because BMI cannot distinguish between people with high muscle mass and those with excess fat. It is increasingly clear that BMI is a rather poor indicator of percent body fat, whereas WC, WHpR, and WHtR are used as surrogate markers for body fat centralization $[5,26]$. WC accurately reflects the degree of visceral fat rather than the absolute degree of adiposity, but it does not take into account height differences and may overestimate or 
Table 3 The difference among the AUC values of baseline indices of obesity in the cross-sectional and cohort studies.

\begin{tabular}{|c|c|c|c|}
\hline Cross-sectional study & $\boldsymbol{N}=1727$ & AUC $(95 \% \mathrm{Cl})$ & $\boldsymbol{P}$-value* \\
\hline Men & $N=768$ & & \\
\hline WHtR - WHpR & & $0.049(0.025-0.072)$ & $<0.001$ \\
\hline WHtR - BMI & & $0.202(0.003-0.050)$ & 0.027 \\
\hline WHpR - BMI & & $-0.022(-0.058-0.014)$ & 0.228 \\
\hline Women & $N=959$ & & \\
\hline WHtR - WHpR & & $0.057(0.036-0.077)$ & $<0.001$ \\
\hline WHtR - BMI & & $0.014(-0.008-0.036)$ & 0.202 \\
\hline WHpR - BMl & & $-0.042(-0.077-0.008)$ & 0.016 \\
\hline Cohort study & $N=419$ & AUC (95\% Cl) & $P$-value* \\
\hline Men & $N=164$ & & \\
\hline WHtR - WHpR & & $0.013(-0.053-0.079)$ & 0.702 \\
\hline WHtR - BMI & & $0.034(-0.030-0.099)$ & 0.297 \\
\hline WHpR - BMI & & $0.021(-0.076-0.119)$ & 0.667 \\
\hline Women & $N=255$ & & \\
\hline WHtR - WHpR & & $0.040(-0.009-0.089)$ & 0.107 \\
\hline WHtR - BMI & & $-0.012(-0.064-0.040)$ & 0.648 \\
\hline WHpR - BMI & & $-0.052(-0.133-0.029)$ & 0.206 \\
\hline
\end{tabular}

AUC area under the curve. * $P$-value: ROC analysis. Bold values indicate significance $(p<0.05)$ underestimate the risk of CVD [12, 27]. Hsieh et al. [12] showed that people with a noticeably large WC may have the same health risks as the above items regardless of their height, but short people have higher health risks than tall people in the moderately large WC population of Japanese men. Of these three measurements, only the WHpR considers differences in body structure and is an anthropometric measure commonly used to characterize regional adiposity.

WHtR is a simple and practical anthropometric index to identify higher metabolic risks in normal and overweight Japanese men and women [12, 28, 29]. Hsieh et al. [29] suggested that the optimal value for WHtR was 0.5 for risk factors defined by the American Heart Association, the National Heart, Lung, and Blood Institute, and the International Diabetes Federation and approximately 0.5 for other risk factors in both genders. For non-overweight Korean adults, a higher incidence of hypertension was observed in the WHtR $\geq 0.5$ group than the WHtR $<0.5$ group [7]. A Chinese study found that a WHtR cutoff value of $\geq 0.5$ identified people with high adiposity and was strongly associated with hypertension [30]. In our study, a positive association between WHtR and the incidence of hypertension was observed in Japanese adults and a WHtR cutoff value of 0.47 to 0.53 may be a better predictor of incident hypertension.

There are several possible research limitations that could affect our study. First, the cross-sectional study

Table 4 Odds ratios and 95\% Cl for hypertension of subjects according to quartiles of baseline WHtR in the cross-sectional and cohort studies

\begin{tabular}{|c|c|c|c|c|c|c|}
\hline & & \multicolumn{5}{|c|}{ Odds ratio $(95 \% \mathrm{Cl})$} \\
\hline & & \multicolumn{5}{|c|}{ Waist-to-height ratio } \\
\hline & & Quartile 1 & Quartile 2 & Quartile 3 & Quartile 4 & \\
\hline & Men & $0.38-0.47$ & $0.47-0.51$ & $0.51-0.54$ & $0.54-0.74$ & \\
\hline & Women & $0.36-0.49$ & $0.49-0.54$ & $0.54-0.58$ & $0.58-0.80$ & \\
\hline Cross-sectional study & $\boldsymbol{N}=1727$ & $\boldsymbol{N}=432$ & $\boldsymbol{N}=431$ & $\boldsymbol{N}=432$ & $\boldsymbol{N}=432$ & $\boldsymbol{P}$-Value \\
\hline \multicolumn{7}{|l|}{ Hypertension } \\
\hline Incidence & & $202(46.8 \%)$ & $254(58.9 \%)$ & $312(72.2 \%)$ & $351(81.3 \%)$ & $<0.001$ \\
\hline Non-adjusted & & 1 & $1.63(1.25-2.14)$ & $2.96(2.23-3.93)$ & $4.93(3.63-6.71)$ & $<0.001$ \\
\hline Gender and age-adjusted & & 1 & $1.55(1.17-2.05)$ & $2.71(2.02-3.63)$ & $4.13(3.01-5.67)$ & $<0.001$ \\
\hline Multivariate-adjusted & & 1 & $1.44(1.07-1.94)$ & $2.35(1.71-3.22)$ & $3.44(2.43-4.88)$ & $<0.001$ \\
\hline Cohort study & $N=419$ & $N=170$ & $N=127$ & $N=79$ & $N=43$ & $P$-Value \\
\hline \multicolumn{7}{|l|}{ Hypertension } \\
\hline Incidence & & 27 (15.9\%) & $30(23.6 \%)$ & $24(30.4 \%)$ & $14(32.6 \%)$ & 0.022 \\
\hline Non-adjusted & & 1 & $1.64(0.92-2.93)$ & $2.31(1.23-4.35)$ & $2.56(1.20-5.46)$ & 0.022 \\
\hline Gender and age-adjusted & & 1 & $1.58(0.88-2.83)$ & $2.13(1.12-4.04)$ & $2.28(1.04-5.00)$ & 0.064 \\
\hline Multivariate-adjusted & & 1 & $1.54(0.82-2.92)$ & $2.43(1.18-5.03)$ & $2.52(1.03-6.20)$ & 0.074 \\
\hline
\end{tabular}

Cl confidence interval. * Multivariate-adjusted for gender, age, smoking status, drinking status, exercise habits, presence of cardiovascular disease, triglycerides, $\mathrm{HDL}$-cholesterol, LDL-cholesterol, use of antidyslipidemic medication, $\mathrm{HbA1c}$, use of antidiabetic medication, eGFR, and serum uric acid. Bold values indicate significance $(p<0.05)$ 
Table 5 Best cutoff values of baseline WHtR to predict hypertension in the cross-sectional and cohort studies

\begin{tabular}{llllllll}
\hline Cross-sectional study & $\boldsymbol{N}=1727$ & Cutoff value & Sensitivity & Specificity & PPV & NPV & Efficiency \\
\hline Men & $N=768$ & 0.5250 & $44.3 \%$ & $80.2 \%$ & $69.1 \%$ & $59.0 \%$ & $62.2 \%$ \\
Women & $N=959$ & 0.5376 & $60.9 \%$ & $68.6 \%$ & $66.0 \%$ & $63.7 \%$ & $64.8 \%$ \\
Cohort study & $\boldsymbol{N}=\mathbf{4 1 9}$ & Cutoff value & Sensitivity & Specificity & PPV & NPV & Efficiency \\
Men & $N=164$ & 0.4701 & $85.4 \%$ & $39.8 \%$ & $58.7 \%$ & $73.2 \%$ & $62.6 \%$ \\
Women & $N=255$ & 0.5136 & $66.7 \%$ & $58.2 \%$ & $61.5 \%$ & $62.6 \%$ & $62.5 \%$ \\
\hline
\end{tabular}

PPV positive predictive value; NPV negative predictive value

design does not establish a cause-and-effect relationship between conventional obesity indices and the presence of hypertension. Second, we must consider the influence that medications for hypertension, dyslipidemia, and hyperglycemia had on the present findings. Third, the measurements of WHtR and confounding factors are based on a single evaluation, which may introduce a misclassification bias. Fourth, the longitudinal analyses were reflected by a relatively smaller sample size and discrepancies in the sequential measurements of confounders in 2014 and 2017. The cohort was slightly younger and healthier compared to participants not included in the longitudinal analyses, which may have caused an underestimation of incident hypertension at the three-year follow-up. Thus, the demographics and referral source may limit the generalizability of the obtained results.

\section{Conclusion}

The findings of this present study suggest that WHtR is strongly associated with the prevalence and incidence of hypertension among Japanese community-dwelling individuals. Thus, WHtR might be an important marker for the assessment of risk and become a therapeutic target for hypertension. For healthy community residents, prospective population-based studies are necessary to investigate interventions such as effective lifestyle improvement and other interventions to control WHtR in adults.

\section{Abbreviations}

BMI: Body mass index; WC: Waist circumference; WHtR: Waist-to-height ratio; WHpR: Waist-to-hip ratio; CVD: Cardiovascular disease; SBP: Systolic blood pressure; DBP: Diastolic blood pressure; TG: Triglycerides; HDL-C: High-density lipoprotein cholesterol; LDL-C: Low-density lipoprotein cholesterol; HbA1c: Hemoglobin A1c; SUA: Serum uric acid; Cr: Creatinine; eGFR: Estimated glomerular filtration ratio; CKD-EPI: Chronic Kidney Disease

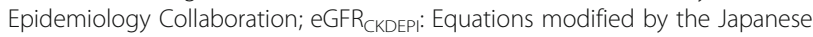
coefficient; SD: Standard deviation; ROC: Receiver operating characteristic; AUC: Area under the ROC curve

\section{Acknowledgments}

We would like to thank Uni-edit (https://uni-edit.net/) for editing and proof reading this manuscript.

\section{Authors' contributions}

RK and TK participated in the design of the study, performed the statistical analysis and drafted the manuscript. RK, TA, AK, and DN contributed to the acquisition and interpretation of data. RK contributed to the conception and design of the statistical analysis. All authors read and approved the manuscript.

\section{Funding}

This work was supported in part by a grant-in-aid from the Foundation for Development of Community (2019). No additional external funding was received for this study. The funders had no role in the study design, data collection and analysis, decision to publish, or preparation of the manuscript.

Availability of data and materials

Not applicable.

Ethics approval and consent to participate

This study was approved by the ethics committee of Ehime University

School of Medicine, and written informed consent was obtained from each subject.

Consent for publication

Not applicable.

\section{Competing interests}

The authors declare that they have no competing interests.

Received: 7 December 2019 Accepted: 13 March 2020

Published online: 15 May 2020

\section{References}

1. Weiss R, Dziura J, Burgert TS, Tamborlane W, Taksali SE, Yeckel CW, et al. Obesity and the metabolic syndrome in children and adolescents. N Engl J Med. 2004;350(23):2362-74.

2. Jiang SZ, Lu W, Zong XF, Ruan HY, Liu Y. Obesity and hypertension. Exp Ther Med. 2016;12(4):2395-9.

3. Nishida C, Ko GT, Kumanyika S. Body fat distribution and noncommunicable diseases in populations: overview of the 2008 WHO expert consultation on waist circumference and waist-hip ratio. Eur J Clin Nutr. 2010;64(1):2-5.

4. Chei $\mathrm{CL}$, Iso H, Yamagishi $\mathrm{K}$, Tanigawa $\mathrm{T}$, Cui $\mathrm{R}$, Imano $\mathrm{H}$, et al. Body fat distribution and the risk of hypertension and diabetes among Japanese men and women. Hypertens Res. 2008;31(5):851-7.

5. Zhou Z, Hu D, Chen J. Association between obesity indices and blood pressure or hypertension: which index is the best? Public Health Nutr. 2009; 12(8):1061-71.

6. Lee JW, Lim NK, Baek TH, Park SH, Park HY. Anthropometric indices as predictors of hypertension among men and women aged 40-69 years in the Korean population: the Korean genome and epidemiology study. BMC Public Health. 2015:15:140.

7. Choi JR, Koh SB, Choi E. Waist-to-height ratio index for predicting incidences of hypertension: the ARIRANG study. BMC Public Health. 2018 ; 18(1):767.

8. Dutra MT, Reis DBV, Martins KG, Gadelha AB. Comparative evaluation of adiposity indices as predictors of hypertension among Brazilian adults. Int J Hypertens. 2018;2018:8396570.

9. Deng G, Yin L, Liu W, Liu X, Xiang Q, Qian Z, et al. Associations of anthropometric adiposity indexes with hypertension risk: a systematic review and meta-analysis including PURE-China. Medicine (Baltimore). 2018; 97(48):e13262.

10. Rothman KJ. BMl-related errors in the measurement of obesity. Int J Obes. 2008;32(Suppl 3):S56-9. 
11. Ortega FB, Sui X, Lavie CJ, Blair SN. Body mass index, the Most widely used but also widely criticized index: would a criterion standard measure of Total body fat be a better predictor of cardiovascular disease mortality? Mayo Clin Proc. 2016;91(4):443-55.

12. Hsieh SD, Yoshinaga H, Muto T. Waist-to-height ratio, a simple and practical index for assessing central fat distribution and metabolic risk in Japanese men and women. Int J Obes Relat Metab Disord. 2003;27(5):610-6.

13. Tuan NT, Adair LS, Stevens J, Popkin BM. Prediction of hypertension by different anthropometric indices in adults: the change in estimate approach. Public Health Nutr. 2010;13(5):639-46.

14. de Oliveira CM, Ulbrich AZ, Neves FS, Dias FAL, Horimoto A, Krieger JE, et al. Association between anthropometric indicators of adiposity and hypertension in a Brazilian population: Baependi heart study. PLoS One. 2017;12(10):e0185225.

15. Abshire DA, Mudd-Martin G, Moser DK, Lennie TA. Comparing measures of general and abdominal adiposity as predictors of blood pressure in college students. J Am Coll Heal. 2018;66(1):51-60.

16. Wu X, Li B, Lin WQ, Huang LL, Wang XX, Fu LY, et al. The association between obesity indices and hypertension: which index is the most notable indicator of hypertension in different age groups stratified by sex? Clin Exp Hypertens. 2019:41(4):373-80.

17. Kawamoto R, Ninomiya D, Kumagi T. Handgrip strength is positively associated with mildly elevated serum bilirubin levels among communitydwelling adults. Tohoku J Exp Med. 2016;240(3):221-6.

18. Kawamoto R, Tabara Y, Kohara K, Miki T, Ohtsuka N, Kusunoki T, et al. Smoking status is associated with serum high molecular adiponectin levels in community-dwelling Japanese men. J Atheroscler Thromb. 2010;17(4): 423-30.

19. Kawamoto R, Tabara Y, Kohara K, Miki T, Ohtsuka N, Kusunoki T, et al. Alcohol drinking status is associated with serum high molecular weight adiponectin in community-dwelling Japanese men. J Atheroscler Thromb. 2010;17(9):953-62.

20. Horio M, Imai E, Yasuda Y, Watanabe T, Matsuo S. Modification of the CKD epidemiology collaboration (CKD-EPI) equation for Japanese: accuracy and use for population estimates. Am J Kidney Dis. 2010;56(1):32-8.

21. Chobanian AV, Bakris GL, Black HR, Cushman WC, Green LA, Izzo JL Jr, et al. The seventh report of the joint National Committee on prevention, detection, evaluation, and treatment of high blood pressure: the JNC 7 report. Jama. 2003;289(19):2560-72.

22. Fluss $R$, Faraggi $D$, Reiser $B$. Estimation of the Youden index and its associated cutoff point. Biom J. 2005;47(4):458-72.

23. Bertsias G, Mammas I, Linardakis M, Kafatos A. Overweight and obesity in relation to cardiovascular disease risk factors among medical students in Crete, Greece. BMC Public Health. 2003:3:3

24. Zhang $M$, Zhao $Y$, Wang $G$, Zhang $H$, Ren $Y$, Wang $B$, et al. Body mass index and waist circumference combined predicts obesity-related hypertension better than either alone in a rural Chinese population. Sci Rep. 2016;6:31935.

25. Janssen I, Heymsfield SB, Wang ZM, Ross R. Skeletal muscle mass and distribution in 468 men and women aged 18-88 yr. J Appl Physiology (Bethesda, Md : 1985). 2000;89(1):81-8.

26. Browning LM, Hsieh SD, Ashwell M. A systematic review of waist-to-height ratio as a screening tool for the prediction of cardiovascular disease and diabetes: 0.5 could be a suitable global boundary value. Nutr Res Rev. 2010; 23(2):247-69.

27. Schneider HJ, Klotsche J, Silber S, Stalla GK, Wittchen HU. Measuring abdominal obesity: effects of height on distribution of cardiometabolic risk factors risk using waist circumference and waist-to-height ratio. Diabetes Care. 2011;34(1):e7.

28. Hsieh SD, Muto T. The superiority of waist-to-height ratio as an anthropometric index to evaluate clustering of coronary risk factors among non-obese men and women. Prev Med. 2005:40(2):216-20.

29. Hsieh SD, Ashwell M, Muto T, Tsuji H, Arase Y, Murase T. Urgency of reassessment of role of obesity indices for metabolic risks. Metabolism. 2010;59(6):834-40.

30. Ren Q, Su C, Wang H, Wang Z, Du W, Zhang B. Prospective study of optimal obesity index cut-off values for predicting incidence of hypertension in 1865-year-old Chinese adults. PLoS One. 2016;11(3):e0148140.

\section{Publisher's Note}

Springer Nature remains neutral with regard to jurisdictional claims in published maps and institutional affiliations.

\section{Ready to submit your research? Choose BMC and benefit from:}

- fast, convenient online submission

- thorough peer review by experienced researchers in your field

- rapid publication on acceptance

- support for research data, including large and complex data types

- gold Open Access which fosters wider collaboration and increased citations

- maximum visibility for your research: over $100 \mathrm{M}$ website views per year

At BMC, research is always in progress.

Learn more biomedcentral.com/submissions 